\title{
An Efficient and Robust Anonymous Authentication Scheme in Global Mobility Networks
}

\author{
Jaecheol Ha* \\ *Department of Information Security, Hoseo University, Korea \\ jcha@hoseo.edu
}

\begin{abstract}
Anonymous user authentication is an essential security mechanism for roaming services in global mobility networks (GLOMONET). Recently, Zhao et al. propose a two-factor anonymous authentication scheme to achieve mutual authentication and session key establishment between the mobile user (MU) and a foreign agent (FA). This paper shows that their scheme has some security vulnerabilities and operational inefficiencies: (1) The scheme fails to protect against a strong replay attack, (2) it is impractical because it requires the predistribution of system parameters, and (3) it is inefficient because of unnecessary encryption/decryption operations. To remedy these weaknesses, this paper proposes a novel anonymous authentication scheme using a smart card. The proposed scheme provides security requirements such as user anonymity, perfect forward secrecy, correct password change, authentication when the MU is located in the home network $(H N)$, and no predistribution of system parameters. In addition, the proposed scheme is secure against various attacks such as impersonation attack, replay attack, off-line password attack, insider attack, stolen-verifier attack, and local password attack. A performance analysis and a comparison with existing schemes show that the proposed scheme is more suitable for low-power and resource-limited mobile environments.
\end{abstract}

Keywords: Mutual authentication, User anonymity, Session key agreemen, Smart card

\section{Introduction}

In a global mobility network (GLOMONET), a mobile user (MU) can access global services through its relationship with the nearest network access point [1]. If the MU wants to roam into a foreign network, mutual authentication between the MU and a foreign agent (FA) is required with the help of the MU's home agent (HA). Many anonymous authentication schemes have been proposed to preserve personal privacy during roaming access to the FA. Such schemes are based mainly on the two-factor authentication technology to enhance the level of security. Here the two-factor usually refers to the password and smart card of the MU [2, 3].

The main security functions of the anonymous authentication scheme are mutual user authentication and session key establishment between the MU and the FA. As pointed out in [4], a strong user authentication scheme should satisfy the following requirements: (1) user anonymity, (2) low communication costs and computational complexity, (3) single registration, (4) update session key periodically, (5) user friendly, (6) no password/verifier table, (7) update password securely and freely, (8) prevention of fraud, (9) prevention of replay attack, (10) security, and (11) the provision of an authentication scheme when the user is located in the home network $(\mathrm{HN})$.

In 2004, Zhu and Ma [5] propose the first anonymous authentication scheme for wireless communications. However, Lee et al. [2] show that their scheme cannot achieve mutual authentication and perfect backward secrecy and that the scheme is vulnerable to the forgery attack and propose an enhanced scheme with minor modifications. Later, Wu et al. [3] and Chang et al. [6] independently find that Lee et al.'s scheme does not provide user anonymity under the forgery attack. Unfortunately, Youn et al. [7] show that both Wu et al. and Chang et 
al.'s schemes do not provide anonymity. In 2011, He et al. [4] demonstrate that Wu et al.'s scheme is vulnerable to several attacks and propose an improved scheme. However, Li and Lee [8] further show that He et al.'s scheme does not satisfy security properties such as user friendliness, fairness in key agreement, and user anonymity. In 2012, Mun et al. [9] analyze Wu et al.'s scheme, pointing out that their scheme also cannot achieve user anonymity, perfect forward secrecy, and the protection of the legal user's password, and then propose an enhanced authentication scheme. Nevertheless, Zhao et al. find that Mun et al.'s scheme is also vulnerable to both impersonation attack and insider attack.

Recently, some new anonymous authentication schemes have been proposed [10-17]. This paper investigates these schemes based on security requirements for secure roaming services in GLOMONET and shows that most have several weaknesses. Unfortunately, the results show that Zhao et al.'s scheme [10] is vulnerable to a strong replay attack and is impractical in terms of the pre-distribution of system parameters. In addition, because their scheme includes useless encryption/decryption operations for open messages, it is inefficient in terms of computational complexity. To overcome these weaknesses and inefficiencies, this paper proposes an effective and secure anonymous authentication scheme designed to satisfy various security requirements and be suitable for low-power and resource-limited mobile devices by reducing computational costs.

The rest of this paper is organized as follows: Section 2 provides some mathematical preliminaries that are useful for a security analysis. Section 3 reviews Zhao et al.'s scheme. Section 4 shows this scheme's security weaknesses and points out its inefficiencies. Section 5 proposes a novel anonymous authentication scheme, and Sections 6 and 7 provide a security analysis of the proposed scheme and a performance comparison with existing schemes, respectively. Section 8 concludes.

\section{Mathematical Preliminaries}

This section discusses the one-way hash function and two computational problems: the discrete logarithm problem (DLP) and the computational Diffie-Hellman problem (CDHP), which are useful for analyzing the security of anonymous authentication schemes described in this paper.

\subsection{One-way Hash Function}

The one-way hash function $h:\{0,1\}^{*} \rightarrow\{0,1\}^{l}$ takes a message of any length as the input and produces a fixed-length output called as a hash value or message digest. A hash function has the following cryptographic properties [18]:

(1) Pre-image resistance: Given a hash value $y$, it should be difficult to find any input message $x$ such that $y=h(x)$. This property is related to that of the one-way function. A hash function that lacks this property is vulnerable to the pre-image attack.

(2) Second pre-image resistance: Given an input $x_{1}$, it should be difficult to find another input $x_{2}$ such that $x_{1} \neq x_{2}$ and $h\left(x_{1}\right)=h\left(x_{2}\right)$. A hash function that lacks this property is vulnerable to a second pre-image attack.

(3) Collision resistance: It should be difficult to find two different input messages $x_{1}$ and $x_{2}$ such that $x_{1} \neq x_{2}$ and $h\left(x_{1}\right)=h\left(x_{2}\right)$. Such an input pair is called a hash collision.

One of the most important cryptographic applications using the one-way hash function is the integrity verification of messages. In addition, the hash function can be applied to generate the message authentication code (MAC) or sign on the message to provide message authentication and non-repudiation services.

\subsection{Discrete Logarithm Problem}

Given elements $g$ and $y$ of the multiplicative group $Z_{p}^{*}$, the discrete logarithm problem (DLP) is the problem of finding a number $x$ such that $g^{x}=y \bmod p$. If $p$ is assumed to be a large integer, then it is 
difficult to determine $x$ given $g$, $p$, and $y$. The difficulty of the discrete logarithm problem is the basis for the security of several cryptographic systems such as the Diffie-Hellman key agreement [19], the digital signature algorithm, and their elliptic curve cryptography analogs [20].

\subsection{Computational Diffie-Hellman Problem}

Given an element $g$ and values of $X=g^{x} \bmod p$ and $Y=g^{y} \bmod p$, the computational DiffieHellman problem (CDHP) is the problem of finding the value $g^{x y} \bmod p$. It is difficult to find $g^{x y} \bmod p$ for given $g, p, X$, and $Y$ if $p$ is a large integer. For example, in the Diffie-Hellman key agreement protocol, even if an attacker eavesdrops $X=g^{x} \bmod p$ and $Y=g^{y} \bmod p$ during the exchange process, the attacker cannot compute the common key $g^{x y} \bmod p$ shared between two parties.

\section{Review of Zhao et al.'s Scheme}

This section reviews Zhao et al.'s anonymous authentication scheme [10]. This scheme consists of four phases: registration phase, authentication and session key establishment phase, update session key phase, and update password phase. This scheme contains the authentication and session key establishment phase when the MU is located in the HN. This paper briefly describes the registration and authentication and session key establishment phases of Zhao et al.'s scheme. Notations and computational operations for a simple description of schemes in this paper are listed in Table 1.

\subsection{Registration Phase}

If the MU wants to be a legal client, then it must first register to its HA through the following steps (Figure 1 details the registration phase):

Step R1: The MU chooses the identity $I D_{M U}$ and password $P W_{M U}$ and generates a random number $N_{M U}$. The MU then sends $I D_{M U}$ and $h\left(P W_{M U} \| N_{M U}\right)$ to the HA through a secure channel.

Step R2: After receiving the registration message from the MU, the HA computes $Q$ $=h\left(I D_{M U} \| y\right) \oplus h\left(P W_{M U} \| N_{M U}\right)$ and $H=h\left(I D_{M U} \| h\left(P W_{M U} \| N_{M U}\right)\right)$, where $y$ is the HA's secret master key. Then the HA stores the information $\left\{Q, H, C, I D_{H A}\right\}$ in a smart card and issues the smart card to the MU through a secure channel.

Step R3: Upon receiving the smart card, the MU inputs the previously generated random number $N_{M U}$ into the smart card

\subsection{Authentication and Session Key Establishment Phase}

If the MU roams into foreign network, it must prove itself to the FA as a legal user and verify the identity of the FA. The authentication and session key establishment phase of Zhao et al.'s scheme is described through the following steps (Figure 2 details this phase):

Table 1. Notations and Computational Operations

\begin{tabular}{|l|l|}
\hline \multicolumn{1}{|c|}{ Notation } & \\
\hline$M U, F A, H A$ & Mobile user, foreign agent, home agent \\
$I D_{X}$ & Identity of an entity $X$ \\
$P W_{X}$ & Password of an entity $X$ \\
$C e r t_{X}$ & Certificate of an entity $X$ \\
$S_{X}$ & Private key of an entity $X$ \\
$P_{X}$ & Public key of an entity $X$ \\
$S K$ & Session key shared between the MU and the FA \\
$P$ & A point of the additive elliptic curve group \\
$T_{X}$ & Time stamp of an entity $X$ \\
\hline
\end{tabular}




\begin{tabular}{|c|c|}
\hline $\begin{array}{l}p, q \\
g \\
a \\
b \\
c \\
E_{X}[\cdot] / D_{X}[\cdot] \\
E S_{X}\{\cdot\} \\
D P_{X}\{\cdot\} \\
G S_{X}(\cdot) \\
V P_{X}(\cdot) \\
\bigoplus \\
h(\cdot) \\
\| \\
=? \\
T R(\cdot)\end{array}$ & $\begin{array}{l}\text { Large primes such that } q \mid p-1 \\
\text { Generator of a multiplicative subgroup with order } q \\
\text { Random number generated by MU for a session } \\
\text { Random number generated by FA for a session } \\
\text { Secret number fixed by HA } \\
\text { Symmetric encryption/decryption using secret key } \\
\text { Asymmetric encryption using private key } \\
\text { Asymmetric decryption using public key } \\
\text { Signature generation using private key } \\
\text { Signature verification using public key } \\
\text { Exclusive-or (XOR) function } \\
\text { One-way hash function, } h:\{0,1\}^{*} \rightarrow\{0,1\}^{l} \\
\text { Concatenation of two inputs } \\
\text { Comparison of two inputs } \\
k \text {-bits (length of } S K \text { ) truncation from least significant bit of the in }\end{array}$ \\
\hline & $\begin{array}{c}\mathrm{HA} \\
{[y, c, C=c P]}\end{array}$ \\
\hline $\begin{array}{l}\text { Gel } \\
I=\end{array}$ & 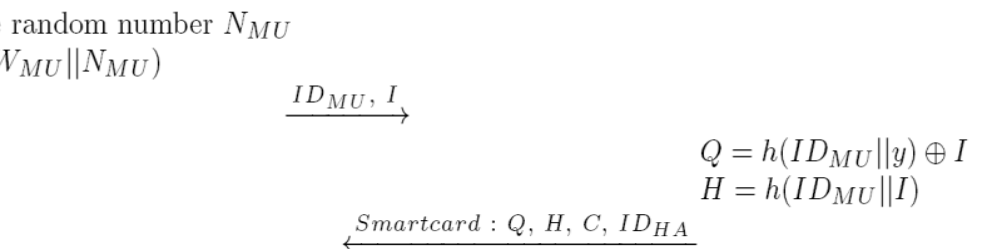 \\
\hline & card : $\left\{Q, H, C, I D_{H A}, N_{M U}\right\}$ \\
\hline
\end{tabular}

Figure 1. Registration Phase of the Zhao et al.'s Scheme

Step A1: The MU inserts its smart card into the card reader and inputs its identity and password. Then the smart card computes $H^{\prime}=h\left(I D_{M U} \| h\left(P W_{M U} \| N_{\mathrm{MU}}\right)\right)$ and checks whether it is equal to $H$. Next, the smart card generates a random number $a$ and computes $A=a P, R_{A C}=a C, N=Q \oplus h\left(P W_{M U} \| N_{M U}\right), D I D_{M U}=I D_{M U} \oplus h\left(R_{A C}\right)$, and $V_{l}=h(N$ $\left.\left\|R_{A C}\right\| I D_{H A}\|A\| C\right)$. Here the computation of $a P$ denotes the scalar multiplication of point $P$ on the cyclic group. Then the MU sends $\left\{A, D I D_{M U}, C, V_{1}, I D_{H A}\right\}$ to the FA through a public wireless channel. 


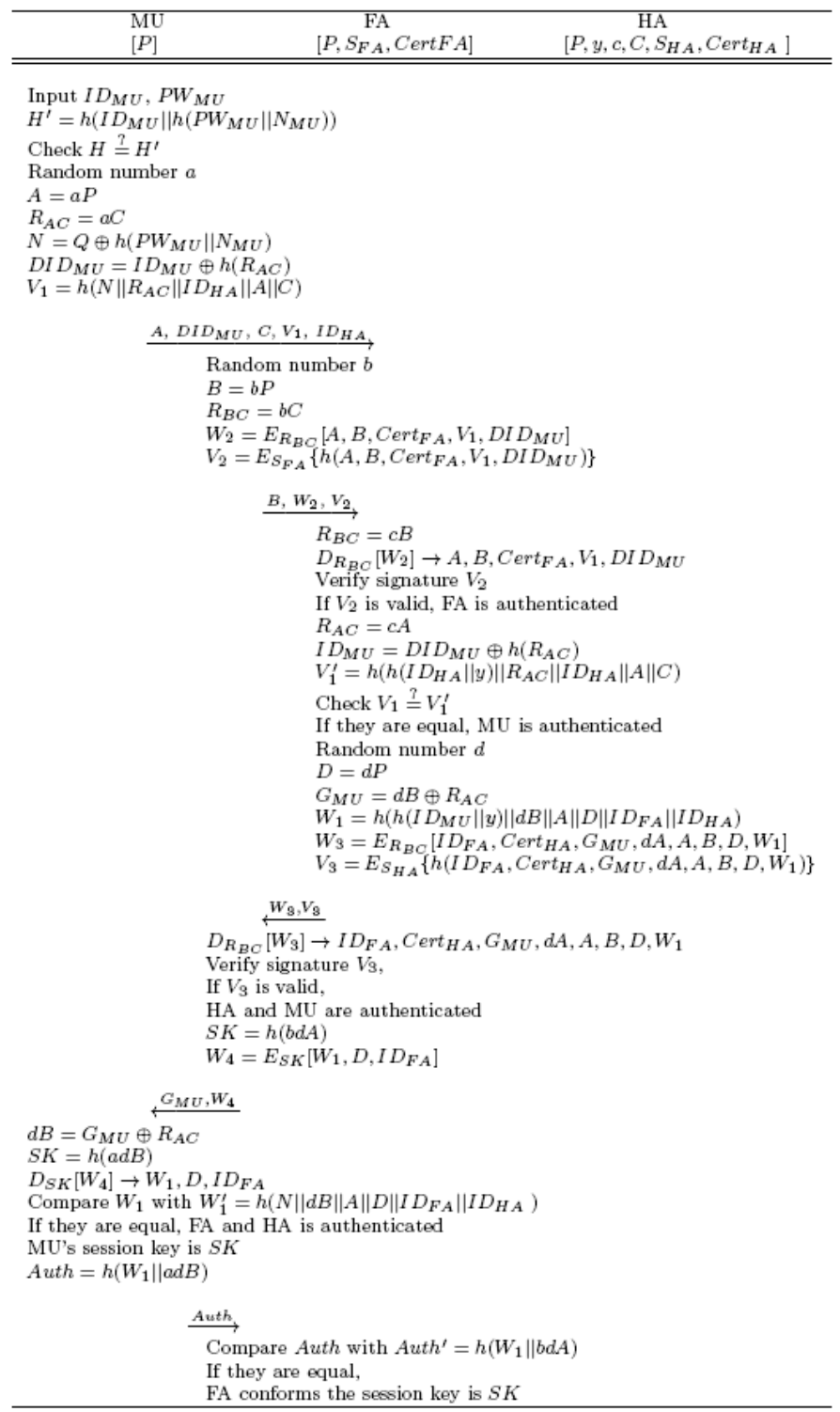

\section{Figure 2. Authentication and Session Key Establishment Phase of the Zhao et al.'s Scheme}

Step A2: After receiving an access request message, the FA generates a random number $b$ and computes $B=b P, R_{B C}=b C, W_{2}=E R_{B C}\left[A, B, C_{C r t}, V_{l}, D I D_{M U}\right]$, and $V_{2}$ $=E S_{F A}\left\{h\left(A, B, \operatorname{Cert}_{F A}, V_{l}, D I D_{M U}\right)\right\}$. Here $S_{F A}$ is a private key of the FA. Then, it sends $\left\{B, W_{2}, V_{2}\right\}$ to the HA.

Step A3: Upon receiving the message from the FA, the HA computes a common key $R_{B C}=c B$ and decrypts $W_{2}$ by using this key. Then, it verifies the FA's signature $V_{2}$ to authenticate the FA by using the certificate $\operatorname{Cert}_{F A}$. Afterward, the HA computes $R_{A C}=c A, I D_{M U}=D I D_{M U} \oplus h\left(R_{A C}\right)$, and $V_{l}{ }^{\prime}=$ $h\left(h\left(I D_{H A} \| y\right)\left\|R_{A C}\right\| I D_{H A}\|A\| C\right)$. Then, to authenticate the MU, it checks whether $V_{I}$ is equal to $V_{l}$ '. Next, the HA generates a random number $d$ and computes $D=d P$, $G_{M U}=d B \oplus R_{A C}, W_{1}=h\left(h\left(I D_{M U} \| y\right)\|d B\| A\|D\| I D_{F A} \| I D_{H A}\right), W_{3}=E R_{B C}\left[I D_{F A}\right.$, $\left.\operatorname{Cert}_{H A}, G_{M U}, d A, A, B, D, W_{l}\right]$, and $V_{3}=E S_{H A}\left\{h\left(I D_{F A}, C \operatorname{Crt}_{H A}, G_{M U}, d A, A, B, D\right.\right.$, $\left.\left.W_{1}\right)\right\}$. Finally, the HA sends $\left\{W_{3}, V_{3}\right\}$ to the FA.

Step A4: After receiving the response message from the HA, the FA computes a common key $R_{B C}=c B$ and decrypts $W_{3}$ by using this key. Then, it verifies the HA's signature $V_{3}$ to authenticate the HA by using the certificate Cert $_{H A}$. After 
authenticating the HA and the MU, the FA computes the session key $S K=h(b d A)$ and $W_{4}=E_{S K}\left[W_{l}, D, I D_{F A}\right]$ and then sends $\left\{G_{M U}, W_{4}\right\}$ to the MU.

Step A5: The MU computes $d B=G_{M U} \oplus R_{A C}$ and decrypts $D_{S K}\left[W_{4}\right]$ by using the session key $S K=h(a d B)$. Then it computes $W_{l}{ }^{\prime}=h\left(N|| d B|| A|| D \| I D_{F A}|| I D_{H A}\right)$ and compares it with $W_{l}$. If they are equal, then the FA and the HA are authenticated. Further, the MU confirms that the SK is the common session key shared with the FA. Finally, the MU computes Auth $=h\left(W_{l} \| a d B\right)$ and sends it to the FA.

Step A6: When the FA receives the message Auth, it computes Auth' $=$ $h\left(W_{l} \| b d A\right)$ and compares it with Auth. If they are equal, then the FA verifies that the $S K$ is the common session key shared with the MU.

\section{Weaknesses and Inefficiencies of Zhao et al.'s Scheme}

This section shows that Zhao et al.'s anonymous authentication scheme has the following weaknesses and inefficiencies:

\subsection{Weaknesses Against the Replay Attack}

In Zhao et al. 's scheme, suppose an attacker may intercept the login request message $\left\{A, D I D_{M U}, C, V_{l}, I D_{H A}\right\}$ and send it to the FA next time. The FA and the HA accept this replay message for anonymous authentication and ensure that the attacker is a legal user. As a result, the attacker receives the response message $\left\{G_{M U}, W_{4}\right\}$ from the FA. Although the attacker cannot compute the session key shared with the FA because it lacks knowledge of the MU's random number $a$, it can impersonate the MU to access the FA. This re play attack obviously causes unnecessary communications and computational costs. Therefore, Zhao et al.'s scheme fails to protect against a strong replay attack.

In addition, it can be assumed that the attack can replay mass login messages to the FA at the same time. Then the FA and the HA should always process from Step A2 to Step A4 per the request message. They cannot detect the attacker's behavior among all access requests in this strong replay attack. Therefore, because the FA and the HA can exhaust their computational resources, the FA or HA in Zhao et al.'s scheme can become a victim by the denial of service (DoS) attack. Because the distributed DoS (DDoS) attack causes the FA or HA to sit down, this replay-based DoS attack can become an important threat in Zhao et al.'s scheme.

\subsection{Pre-distribution of System Parameters}

The cryptographic primitive such as the hash function or encryption does not need any extra system parameters. However, in the primitive such as the Diffie-Hellman key agreement or digital signature, which is based on public key cryptography, system parameters should be handed over to the other entity through the pre-distribution or transmission during the communication session. All entities in Zhao et al.'s scheme uses the scalar point $P$ and elliptic curve system parameters such as the field prime and coefficients of the curve equation. However, no entity transmits these system parameters in all transactions. This means that a trusted third party at the top level is required to provide these system parameters. This party should generate these system parameters once and previously distribute them to all HAs and FAs. It is extremely inconvenient when a new FA or HA joins GLOMONET. Further, because all entities should compulsorily use the same system parameters, Zhao et al.'s scheme is rigid and impractical. Therefore, there is a need for a more flexible and diverse anonymous authentication scheme. Under the assumption that GLOMONET contains various HAs, it should at least consider that the HA may generate different system parameters with other HAs and store them in the smart card of its MU. 


\subsection{Inefficiencies from Unnecessary Encryptions}

In Zhao et al.'s scheme, the FA has two encryptions for the message, and the HA has one. In addition, the MU, the FA, and the HA all have a decryption operation according to the encrypted message respectively. The encryption function is basically performed to provide message confidentiality. However, most messages used as an encryption input are transmitted through networks in their scheme. Further, the certificate of the FA or HA is generally open information.

For example, inputs of the encrypted output $W_{2}$ are $A, B, \operatorname{Cert}_{F A}, V_{1}$, and $D I D_{M U}$. Unfortunately, these inputs are known to all entities, including malicious attackers. Therefore, the encryption of a known message is unnecessary with respect to the cryptographic service of confidentiality. Among input messages of an encryption, only the $d A$ value is not revealed. Despite this fact, even if the $d A$ value is opened, it does not matter to security. As a result, because there is no need to hide sending messages in Zhao et al.'s scheme, encryption and decryption operations that increase operational complexity are useless.

\section{The Proposed Scheme}

This section proposes a lightweight and robust anonymous authentication scheme for roaming services in GLOMONET. The design elements of this proposed scheme include computational efficiency and resistance to security threats. The HA chooses a multiplication group $G$ and a generator $g \in G$ whose order is $q$. That is, $q$ is the smallest positive integer satisfying $g^{q} \bmod p=1$, where $p$ and $q$ are large primes. In addition, $p$ is the modulus for operations in group $G$ such as $q \mid p-1$. The HA first chooses a random secret number $c(<q)$ and computes $C=g^{c} \bmod p$. In addition, the HA has a private/public key pair $S_{H A} / P_{H A}$ and the certificate $\operatorname{Cert}_{H A}$ of its public key. The FA also has its private/public key pair and certificate.

\subsection{Security Model in GLOMONET}

To design a practical and secure anonymous authentication scheme, security functions of each entity are investigated. The MU subscribes to its HA as a client. In the registration step, the MU sends its identity and some related messages to the HA and receives a smart card including secret information and system parameters generated by the HA. Afterward, if the MU wants to access the foreign network by using the password and smart card, the HA anonymously authenticates the MU. The HA then informs the FA that the MU is a legal user. Here there is a need for a countermeasure to check replay messages. The use of a time stamp for filtering old messages is a potential solution.

Based on the public key infrastructure (PKI), the FA and the HA mutually authenticate by using certificates of their public key. In addition, the Diffie-Hellman key agreement technique is used to establish a session key between the MU and the FA and authenticate each other. The overall outline of the security model of the proposed scheme is shown in Figure 3. In this GLOMONET environment, there are various HAs and FAs, and the HA has its own system parameters. Therefore, the MU with system parameters of its HA must send them to the FA to access the foreign network.

The proposed scheme consists of five phases: (1) registration phase, (2) authentication and session key establishment phase, (3) password change phase, (4) update session key phase, and (5) authentication and session key establishment phase when the MU is located in its $\mathrm{HN}$. 


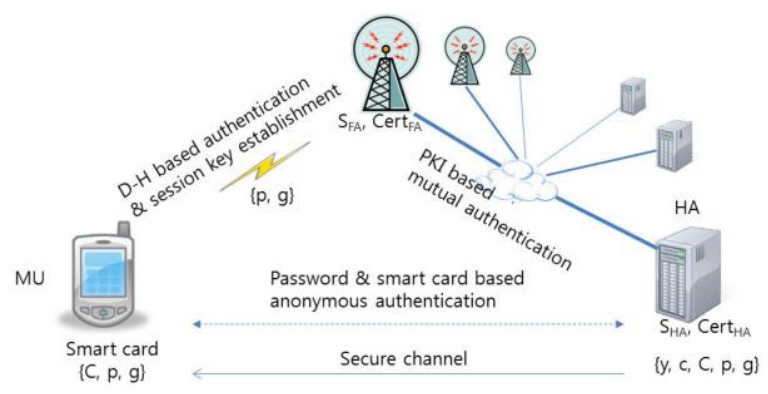

Figure 3. Security Model in GLOMONET

\subsection{Registration Phase}

If the MU wants to register to its HA, it must submit necessary information through a secure channel. After verifying that the MU is legal, the HA issues a temper-resistant smart card to the MU. Figure 4 details the registration phase.

Step R1: A new MU chooses its identity $I D_{M U}$ and password $P W_{M U}$. Then the MU submits its identity $I D_{M U}$ to its HA for registration over a secure channel.

Step R2: After receiving the identity $I D_{M U}$ from the MU, the HA computes $R=$ $h\left(y|| I D_{H A}|| I D_{M U}\right)$, where $y$ is the HA's secret master key. Then the HA stores the information $\left\{R, I D_{H A}, C, g, p\right\}$ in a temper-resistant smart card and issues the smart card to the MU through a secure channel.

Step R3: When receiving the smart card from the HA, the MU generates a large random number $N_{M U}$ and computes $I=h\left(I D_{M U}\left\|P W_{M U}\right\| N_{M U}\right)$ and $H=R \oplus I$. Here the choice space of $I D_{M U}$ and $P W_{M U}$ must be enough to resist a simultaneous guessing attack even when $I$ and $N_{M U}$ are revealed by a physical breach attack on the smart card. Then, the MU replaces $R$ with $H$ in the smart card. In addition, the smart card stores $I$ and $N_{M U}$.

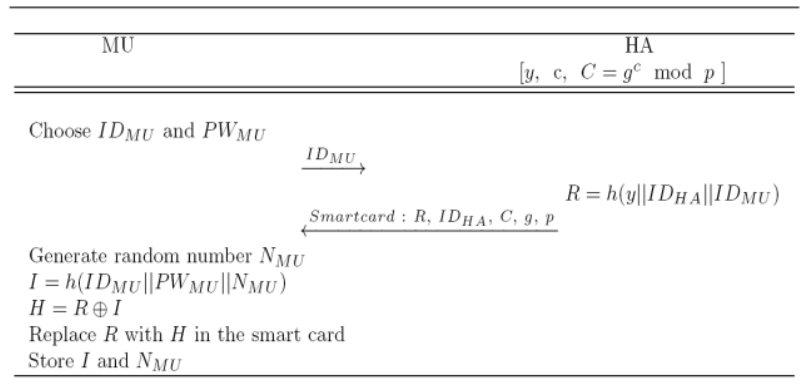

\section{Figure 4. Registration Phase of the Proposed Scheme}

\subsection{Authentication and Session Key Establishment Phase}

If the MU roams into a foreign network, it must authenticate the FA and prove its legality to the FA with the help of its HA. Figure 5 details this phase. The authentication and session key establishment phase between the MU and the FA is described as follows:

Step A1: The MU inserts the smart card into the card reader and enters its identity $I D_{M U}$ and password $P W_{M U}$. After reading $N_{M U}$, the smart card computes $I^{\prime}=h\left(I D_{M U}\right.$ $\left.\left\|P W_{M U}\right\| N_{M U}\right)$ and checks whether $I=I^{\prime}$ by using the stored value $I$. If the checking holds, then the MU is verified to be a legitimate user. Otherwise, this phase is terminated. The smart card then generates a random number $a$ and computes $A^{a} \bmod p, K=C^{a} \bmod p$, $P=I D_{M U} \oplus h\left(K \| T_{M U}\right)$, and $Q=H \oplus I \oplus h(A \| K)$. Finally, the MU sends the necessary message $\left\{A, P, Q, g, p, I D_{H A}, T_{M U}\right\}$ to the FA over a wireless channel. Here, to avoid the user tracking attack, the length of $I D_{M U}$ should be less than $h\left(K \| T_{M U}\right)$.

Step A2: After receiving the request message from the MU, the FA checks the time stamp $T_{M U}$ to determine whether it is within the expected time interval. If not, the access 
request is rejected immediately. Otherwise, the FA keeps the time stamp and the message pair for some time to check the replay attack. If the FA receives the same time stamp and message pairs during this time interval, it decides that the second message is a retransmitted one and discards it. Next, the FA generates a random number $b$ and computes $B=g^{b} \bmod p$. Then it makes a signature by using its private key $S_{F A}$ such that $U$ $=G S_{F A}\left(A, B, P, Q, I D_{F A}, T_{F A}\right)$. Finally, the FA sends $\left\{A, B, P, Q, U, T_{M U}, T_{F A}, I D_{F A}\right.$, $\left.\operatorname{Cert}_{F A}\right\}$ to the HA.

Step A3: If the HA receives the message from the FA, it checks the time stamps $T_{F A}$ and $T_{M U}$ to determine whether they are within the expected time interval and keeps the time stamp and the message pair for some time to check the replay attack. Then the HA verifies the FA's signature $U$ by using the certificate $\operatorname{Cert}_{F A}$ for the FA's public key. If the signature is valid, then the FA is authenticated. Afterward, the HA computes $K^{\prime}=A^{c}$ mod $p, I D_{M U}=P \oplus h\left(K^{\prime} \| T_{M U}\right)$, and $V=h\left(y\left\|I D_{H A}\right\| I D_{M U}\right)$ and then checks whether the value of $Q \oplus h\left(A \| K^{\prime}\right)$ is equal to $V$. If it is valid, then the MU is authenticated. Then the HA stores $A$ and $B$ in $D$ and $E$, respectively, and computes $Y=h\left(V\|D\| B \| I D_{H A}|| I D_{F A}\right)$. Finally, the HA generates a signature by using its private key such that $Z=G S_{H A}\left(Y, D, E, I D_{H A}, I D_{F A}\right)$ and sends $\left\{Y, Z, D, E, I D_{H A}, C_{e r t}\right\}$ to the FA.

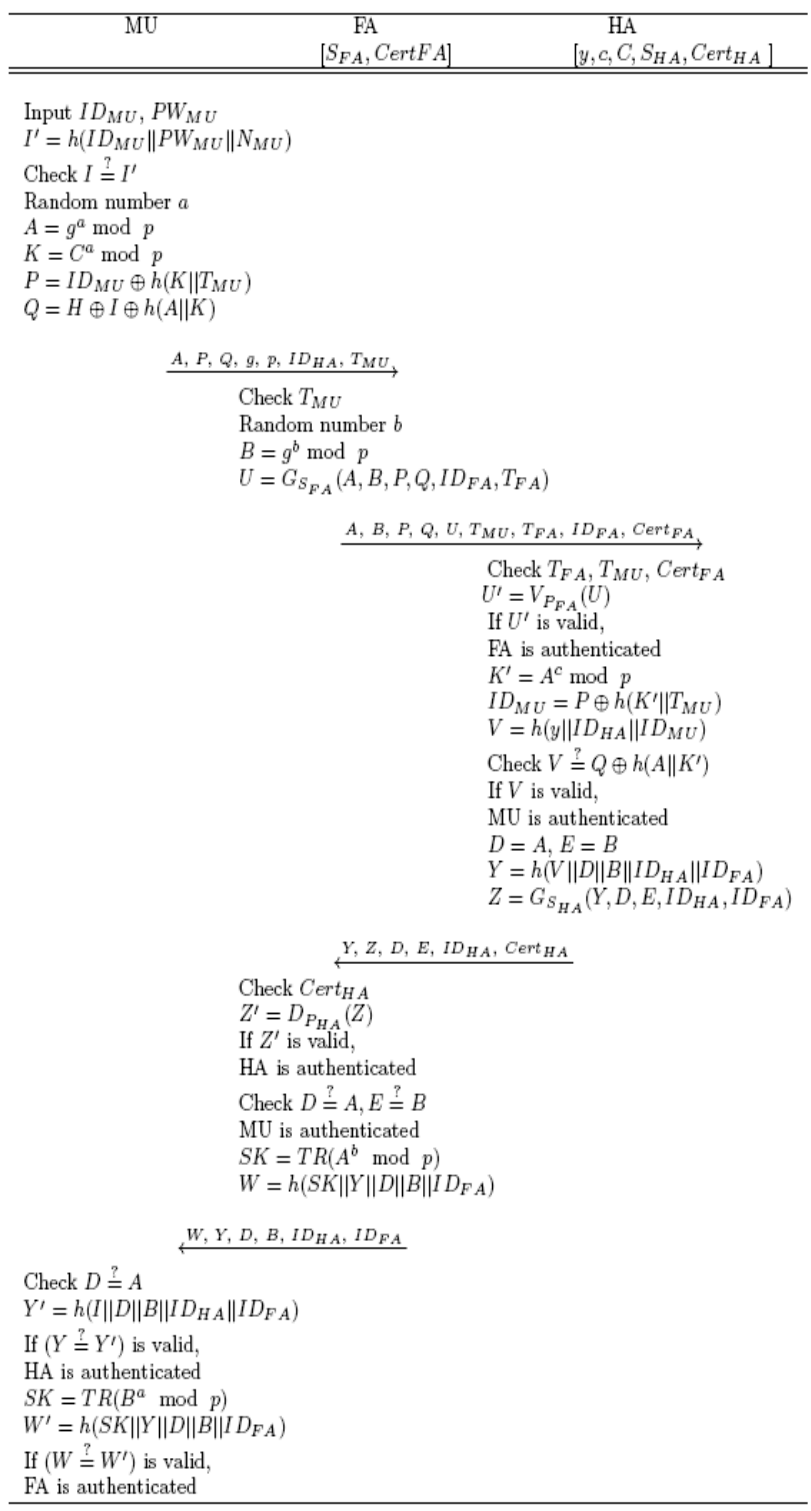




\section{Figure 5. Authentication and Session ey establishment phase of the proposed scheme}

Step A4: After receiving the message from the HA, the FA verifies the HA's signature $Z$ by using the certificate $\operatorname{Cert}_{H A}$ for the HA's public key. If it is valid, then the HA is authenticated. Then the FA checks $D$ and $E$ to determine whether they are the same as $A$ and $B$, respectively. If they are valid, then the MU is authenticated. The FA then computes the session key $S K=T R\left(A^{b} \bmod p\right)$ and makes a message authentication code such that $W$ $=h\left(S K\|Y\| D\|B\| I D_{F A}\right)$. Finally, the FA sends $\left\{W, Y, D, B, I D_{H A}, I D_{F A}\right\}$ to the MU.

Step A5: Upon receiving the message from the FA, the MU checks that the received $D$ is equal to $A$. Then it verifies the message authentication code $Y$ generated by the HA. Then the MU computes the session key $S K=T R\left(B^{a} \bmod p\right)$ and verifies a message authentication code $W$ generated by the FA. If it is valid, then the shared session key $S K=$ $\operatorname{TR}\left(B^{a}=g^{a b} \bmod p\right)$ is verified.

\subsection{Password Change Phase}

This phase occurs when the MU wants to change its password. There is no communication with a foreign network or its HA. The password change phase consists of the following steps:

Step P1: The MU inserts its smart card into the card reader and enters its identity $I D_{M U}$ and old password $P W_{M U}{ }^{\text {old }}$. The smart card computes $I^{\prime}=h\left(I D_{M U}\left\|P W_{M U}{ }^{\text {old }}\right\| N_{M U}\right)$, and checks whether $I=I^{\prime}$ by using the stored value $I$. If it holds, then the MU is verified to be a legitimate user. Otherwise, this phase terminates immediately.

Step P2: The MU generates a new random number $N_{M U}^{\text {new }}$ and inputs a new password $P W_{M U}{ }^{\text {new }}$. Then the smart card computes $I^{\text {new }}=h\left(I D_{M U}\left\|P W_{M U}{ }^{\text {new }}\right\| N_{M U}{ }^{\text {new }}\right)$ and $H^{\text {new }}=H$ $\bigoplus I \oplus I^{\text {new }}$. Finally, the smart card replaces old $H, I$, and $N_{M U}$ values with new values of $H^{\text {new }}, I^{\text {new }}$, and $N_{M U}^{\text {new }}$, respectively.

\subsection{Update Session Key Phase}

The MU and the FA must renew the session key to ensure strong security when the MU is always within the same foreign network. The following processes are used to update the session key in the $i$-th session:

Step U1: The MU selects a random number $a_{i}$, computes $A_{i}=g^{a_{i}} \bmod p$, and sends $A_{i}$ to the FA.

Step U2: After receiving $A_{i}$ from the MU, the FA selects a random number $b_{i}$, computes a new session key $S K_{i}=T R\left(A^{b_{i}} \bmod p\right)$ and $B_{i}=g^{b_{i}} \bmod p$, and then sends $H_{i}=$ $h\left(S K_{i}|| S K_{i-1}\right)$ and $B_{i}$ to the MU.

Step U3: The MU computes a new session key $S K_{i}=T R\left(B^{a_{i}} \bmod p\right)$ and $H_{i}{ }^{\prime}=$ $h\left(S K_{i}|| S K_{i-1}\right)$ and compares $H_{i}$ with $H_{i}{ }^{\prime}$. If they are equal, then the MU verifies the session key. Otherwise, the MU aborts updating the session key.

\subsection{Authentication and Session Key Establishment Phase between the MU and the HA}

If the MU roams into its $\mathrm{HN}$, it must directly authenticate the HA and prove its legality to the HA. Figure 6 provides a detailed description of this phase. The authentication and session key establishment phase when the MU is located in its $\mathrm{HN}$ is described as follows:

Step H1: The MU inserts the smart card into the card reader and enters its identity $I D_{M U}$ and password $P W_{M U}$. After reading $N_{M U}$, the smart card computes $I^{\prime}=h\left(I D_{M U}\right.$ $\left.\left\|P W_{M U}\right\| N_{M U}\right)$ and checks whether $I=I^{\prime}$ by using the stored value $I$. If the checking holds, then the MU is verified to be a legitimate user. Otherwise, this phase is terminated. The smart card then generates a random number a and computes $A=g^{a} \bmod p, K=T R\left(C^{a}\right.$ 
$\bmod p), P=I D_{M U} \oplus h\left(K \| T_{M U}\right)$, and $Q=H \oplus I \oplus h(A \| K)$. Finally, the MU sends the necessary message $\left\{A, P, Q, g, p, I D_{H A}, T_{M U}\right\}$ to the HA over a wireless channel.

Step H2: If the HA receives the message from the MU, it checks the time stamp $T_{M U}$ to determine whether they are within the expected time interval and keeps the time stamp and the message pair for some time to check the replay attack. The HA computes $K^{\prime}=A^{c}$ $\bmod p, I D_{M U}=P \oplus h\left(K^{\prime} \| T_{M U}\right)$, and $V=h\left(y \| I D_{H A}|| I D_{M U}\right)$ and then checks whether the value of $Q \oplus h\left(A \| K^{\prime}\right)$ is equal to $V$. If it is valid, then the MU is authenticated. Then the HA stores $A$ and $K^{\prime}$ in $D$ and $S K$, respectively, and computes $Y=h\left(S K|| D\|C\| I D_{H A}\right)$. Finally, it sends $\left\{Y, D, I D_{H A}\right\}$ to the MU.

Step H3: Upon receiving the message from the HA, the MU checks whether the received $D$ is equal to $A$. Then it verifies the message authentication code $Y$ generated by the HA by using the session key $S K=K$. If it is valid, then the shared session key $S K=$ $T R\left(C^{a}=g^{a c} \bmod p\right)$ is verified.

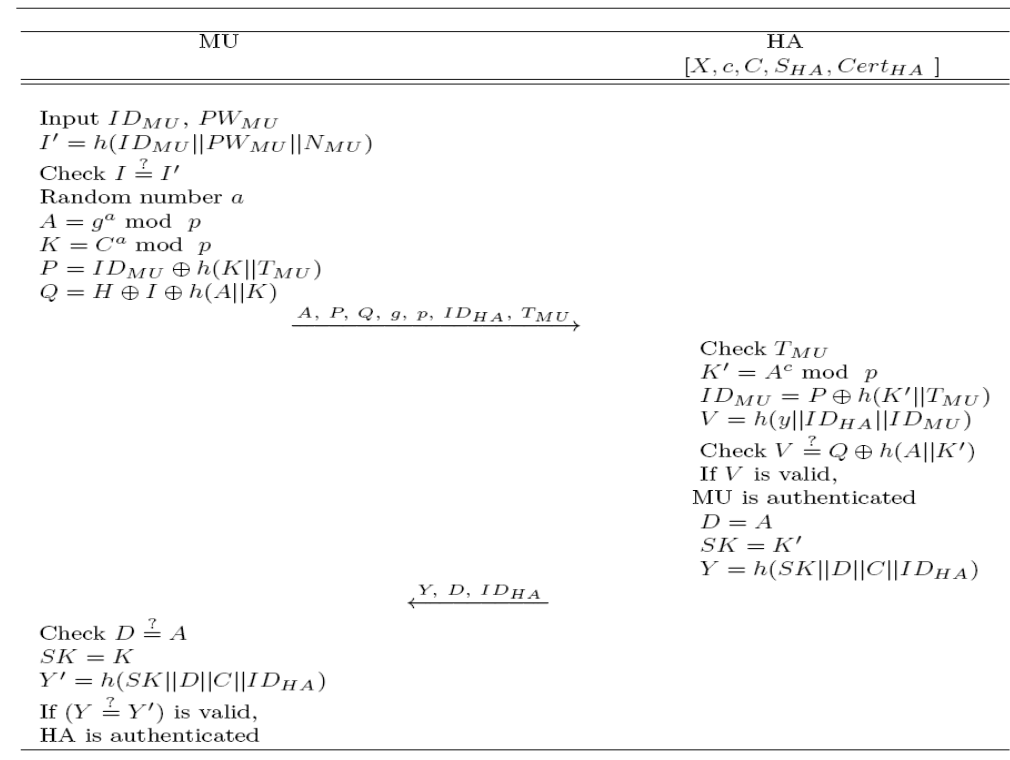

Figure 6. Authentication and Session Key Establishment between MU and HA

\section{Security Analysis}

This section analyzes the security of the proposed scheme according to security requirements for a secure GLOMONET environment and compares the scheme with existing schemes.

\subsection{User Anonymity and Untraceability}

In the proposed scheme, the identity of $\mathrm{MU}, I D_{M U}$, is submitted to the HA over a secure channel in the registration phase. Because only the HA knows the identity of the MU at that time, an attacker cannot obtain it. Assume that any attacker eavesdrops on the message $\left\{A, P, Q, g, p, I D H A, T_{M U}\right\}$ transmitted between the MU and the FA. However, the attacker cannot derive the real identity of the MU without knowing the common secret information $K$ between the MU and the HA. In addition, because the transmitted messages $A, P$ and $Q$ are dynamically changed in each session, the attacker cannot trace the MU's moving history and current location. Therefore, the proposed scheme can provide user anonymity and untraceability for the MU. 


\subsection{Impersonation Attack}

To impersonate as a legal MU, an attacker with some arbitrary $I D_{M U}$ must cheat the HA. In the proposed scheme, the HA authenticates the MU by comparing the value $V=$ $h\left(y|| I D_{H A}|| I D_{M U}\right)$ with $Q \oplus h(A \| K)$. An attacker can arbitrary compute $A$ and $K$ by using its random number a before sending the request message to the FA. However, the attacker does not know the MU's password $P W_{M U}$ and thus cannot compute $I=h\left(I D_{M U} \| P W_{M U}\right.$ $\left.\| N_{M U}\right)$ and $Q \oplus h(A \| K)=H \oplus I$. Therefore, an attacker without knowledge of the MU's password cannot cheat the HA by pretending to be as a legal user. The attacker cannot impersonate as an HA (resp. FA) because messages generated by the attacker without knowledge of the private key are detected by the mutual authentication process of the FA (resp. HA). As a result, the proposed scheme can prevent these impersonation attacks.

\subsection{Replay Attack}

The proposed scheme adopts the time stamp to prevent the replay attack. If an attacker sends the previous session messages $\left\{A, P, Q, g, p, I D_{H A}, T_{M U}\right\}$ to the FA, the FA checks whether the MU's time stamp is within the expected time interval. It is the first countermeasure against the replay attack. Nevertheless, an attack can send the previous message $\left\{A, P, Q, g, p, I D_{H A}, T_{M U}^{c}\right\}$ only by changing the old time stamp $T_{M U}$ to the current one $T_{M U}{ }^{c}$. However, the HA can detect the invalidity of time by computing $I D_{M U}{ }^{*}=P \oplus h\left(K^{\prime} \| T_{M U}{ }^{c}\right)$ and $V^{*}=h\left(y\left\|I D_{H A}\right\| I D_{M U}{ }^{*}\right)$ and checking whether $V^{*}$ is not equal to $Q \oplus h\left(A \| K^{\prime}\right)$. Actually, both $I D_{M U}{ }^{*}$ and $V^{*}$ computed by the HA are incorrect. Consequently, an attacker cannot modify the MU's time stamp without knowing the secret common information $K$ and the identity of the MU. In addition, the time stamp of the FA, $T_{F A}$, can be authenticated to the HA by using the FA's signature $U$. Therefore, the proposed scheme can prevent the replay attack by using the time stamp.

\subsection{Perfect Forward Secrecy}

Perfect forward secrecy means that even if an attacker compromises the previous session key between the MU and the FA, it cannot derive a new session key. In the proposed scheme, because the session key $S K=g^{a b} \bmod p$ is computed using one-time random numbers $a$ and $b$ in each session, an attacker cannot retrieve the session key from transmitted messages $A=g^{a} \bmod p$ and $B=g^{b} \bmod p$ based on the security of the DLP and the CDHP. Because two session keys are always generated independently, knowledge of the previous session key $S K^{*}=g^{a^{*} b^{*}}$ mod $p$ and transmitted messages does not help in deriving the current session key $S K=g^{a b} \bmod p$ and vice versa. Therefore, the proposed scheme can achieve perfect forward secrecy.

\subsection{Off-line Password Guessing Attack}

In the proposed scheme, even when the smart card has temper-resistant protection, it is assumed that the MU's smart card is stolen by an attacker. In addition, the attacker can obtain $H, I, I D_{H A}, C, g, p$, and $N_{M U}$ from the MU's stolen smart card by using strong physical attacks. After guessing the MU's password, the attacker tries to verify that the guessed password is correct or find $I D_{M U}$ by using the aforementioned collected information. The attacker tries to compute $h\left(I D_{M U}\left\|P W_{M U}^{\text {guessed }}\right\| N_{M U}\right)$ and compare it with $I$. However, this is not possible because $I D_{M U}$ is kept only by the MU and the HA. In addition, simultaneous off-line guessing for both the password and identity of the MU is really a hard problem for the attacker. As a result, the proposed scheme is secure against the off-line password guessing attack. 


\subsection{Insider Attack}

If an insider of the HA obtains the MU's password, it can try to impersonate as a legal MU to access the FA. In the registration and authentication phases, the HA knows only the identity of the MU, $I D_{M U}$. However, the insider cannot derive or guess the MU's password without $N_{M U}$. In addition, the MU can change the password $P W_{M U}$ and the random number $N_{M U}$ without the help of the HA in the password change phase. As a result, the proposed scheme can withstand the insider attack.

\subsection{Stolen-Verifier Attack}

The HA and the FA do not maintain any verifier tables and secret key databases. Therefore, the stolen-verifier attack does not apply to the proposed scheme.

\subsection{Local Password Attack}

In the authentication and key establishment phase, if the validity of the user's identity and password is not checked before accessing the FA, then an attacker with a stolen smart card can impersonate a legal user in all transactions. Based on this fact, the proposed scheme is designed to avoid any unauthorized use for mobile devices by verifying the local password. Table 2 compares the proposed scheme with some recently proposed schemes in terms of security properties and functionalities. The proposed scheme satisfies almost all security requirements of GLOMONET.

Table 2. Comparison of Security and Functionality

\begin{tabular}{|c|c|c|c|c|c|}
\hline Security and functionality & $\begin{array}{c}\text { Proposed } \\
\text { scheme }\end{array}$ & $\begin{array}{c}\text { Zhao et al. } \\
{[10]}\end{array}$ & $\begin{array}{c}\text { Xie et al. } \\
{[12]}\end{array}$ & $\begin{array}{c}\text { Das [13] } \\
{[4]}\end{array}$ \\
\hline User anonymity & $\mathrm{O}$ & $\mathrm{O}$ & $\mathrm{O}$ & $\mathrm{O}$ & $\mathrm{X}$ \\
\hline Impersonation attack & $\mathrm{O}$ & $\mathrm{O}$ & $\mathrm{O}$ & $\mathrm{O}$ & $\mathrm{O}$ \\
\hline Strong replay attack & $\mathrm{O}$ & $\mathrm{X}$ & $\mathrm{O}$ & $\mathrm{O}$ & $\mathrm{O}$ \\
\hline Perfect forward secrecy & $\mathrm{O}$ & $\mathrm{O}$ & $\mathrm{O}$ & $\mathrm{O}$ & $\mathrm{X}$ \\
\hline Off-line password guessing attack & $\mathrm{O}$ & $\mathrm{O}$ & $\mathrm{O}$ & $\mathrm{O}$ & $\mathrm{O}$ \\
\hline Insider attack & $\mathrm{O}$ & $\mathrm{O}$ & $\mathrm{O}$ & $\mathrm{O}$ & $\mathrm{O}$ \\
\hline Stolen-Verifier attack & $\mathrm{O}$ & $\mathrm{O}$ & $\mathrm{X}$ & $\mathrm{O}$ & $\mathrm{O}$ \\
\hline Local password attack & $\mathrm{O}$ & $\mathrm{O}$ & $\mathrm{X}$ & $\mathrm{O}$ & $\mathrm{O}$ \\
\hline Authentication when MU is located in HN & $\mathrm{O}$ & $\mathrm{O}$ & $\mathrm{X}$ & $\mathrm{X}$ & $\mathrm{O}$ \\
\hline No pre-distribution of system parameters & $\mathrm{O}$ & $\mathrm{X}$ & $\mathrm{X}$ & $\mathrm{X}$ & $\mathrm{O}$ \\
\hline
\end{tabular}

Note : $\mathrm{O}$ : secure or support $\times$ : insecure or not support

\section{Performance Analysis}

This section compares the performance of the proposed scheme with that of some existing schemes. Here the analysis focuses on the computational cost of the authentication and key establishment phase because this phase is the main part of the anonymous authentication scheme. Table 3 provides the comparison results for the total computational cost. The time complexity of the following primitives is compared in this table: (1) modular exponentiation, (2) scalar multiplication, (3) signature generation, (4) signature verification, (5) symmetric encryption, (6) symmetric decryption, (7) asymmetric encryption, (8) asymmetric decryption, and (9) hash operations.

Table 3. Comparison of the Computational Cost

\begin{tabular}{|c|c|c|c|c|c|c|}
\hline Primitives & Entities & $\begin{array}{c}\text { Proposed } \\
\text { scheme }\end{array}$ & $\begin{array}{c}\text { Zhao et al. } \\
{[10]}\end{array}$ & $\begin{array}{c}\text { Xie et al. } \\
{[12]}\end{array}$ & Das [13] & $\begin{array}{c}\text { He et al. } \\
{[4]}\end{array}$ \\
\hline
\end{tabular}




\begin{tabular}{|c|c|c|c|c|c|c|}
\hline Modular exponentiation & $\begin{array}{c}\text { MU } \\
\text { FA } \\
\text { HA }\end{array}$ & $\begin{array}{l}3 \\
2 \\
1\end{array}$ & $\begin{array}{l}- \\
- \\
-\end{array}$ & $\begin{array}{l}3 \\
2 \\
2\end{array}$ & $\begin{array}{l}4 \\
5 \\
3\end{array}$ & $\begin{array}{l}- \\
- \\
-\end{array}$ \\
\hline Scalar multiplication & $\begin{array}{c}\text { MU } \\
\text { FA } \\
\text { HA }\end{array}$ & $\begin{array}{l}- \\
- \\
-\end{array}$ & $\begin{array}{l}3 \\
3 \\
4\end{array}$ & $\begin{array}{l} \\
- \\
-\end{array}$ & $\begin{array}{l}- \\
- \\
-\end{array}$ & $\begin{array}{l}- \\
- \\
-\end{array}$ \\
\hline Signature generation & $\begin{array}{c}\text { MU } \\
\text { FA } \\
\text { HA }\end{array}$ & $\begin{array}{l}- \\
1 \\
1\end{array}$ & $\begin{array}{l}- \\
1 \\
1\end{array}$ & $\begin{array}{l}- \\
- \\
-\end{array}$ & $\begin{array}{l}- \\
1 \\
1\end{array}$ & $\begin{array}{l}- \\
1 \\
1\end{array}$ \\
\hline Signature verification & $\begin{array}{c}\text { MU } \\
\text { FA } \\
\text { HA }\end{array}$ & $\begin{array}{l}- \\
1 \\
1\end{array}$ & $\begin{array}{l}- \\
1 \\
1\end{array}$ & $\begin{array}{l}- \\
- \\
-\end{array}$ & $\begin{array}{l}- \\
1 \\
1\end{array}$ & $\begin{array}{l}- \\
1 \\
1\end{array}$ \\
\hline Symmetric encryption & $\begin{array}{c}\text { MU } \\
\text { FA } \\
\text { HA }\end{array}$ & $\begin{array}{l}- \\
- \\
-\end{array}$ & $\begin{array}{l}- \\
2 \\
1\end{array}$ & $\begin{array}{l}1 \\
1 \\
1\end{array}$ & $\begin{array}{l}3 \\
2 \\
1\end{array}$ & $\begin{array}{l}1 \\
1 \\
-\end{array}$ \\
\hline Symmetric decryption & $\begin{array}{c}\text { MU } \\
\text { FA } \\
\text { HA }\end{array}$ & $\begin{array}{l}- \\
- \\
-\end{array}$ & $\begin{array}{l}1 \\
1 \\
1\end{array}$ & $\begin{array}{l}1 \\
- \\
3\end{array}$ & $\begin{array}{l}1 \\
2 \\
4\end{array}$ & $\begin{array}{l}1 \\
- \\
2\end{array}$ \\
\hline Asymmetric encryption & $\begin{array}{c}\text { MU } \\
\text { FA } \\
\text { HA }\end{array}$ & $\begin{array}{l}- \\
- \\
-\end{array}$ & $\begin{array}{l}- \\
- \\
-\end{array}$ & $\begin{array}{l}- \\
- \\
-\end{array}$ & $\begin{array}{l}- \\
- \\
-\end{array}$ & $\begin{array}{l}- \\
- \\
1\end{array}$ \\
\hline Asymmetric decryption & $\begin{array}{c}\text { MU } \\
\text { FA } \\
\text { HA }\end{array}$ & $\begin{array}{l}- \\
- \\
-\end{array}$ & $\begin{array}{l}- \\
- \\
-\end{array}$ & $\begin{array}{l} \\
- \\
-\end{array}$ & $\begin{array}{l}- \\
- \\
-\end{array}$ & $\begin{array}{l}- \\
1 \\
-\end{array}$ \\
\hline Hash operation & $\begin{array}{c}\text { MU } \\
\text { FA } \\
\text { HA }\end{array}$ & $\begin{array}{l}5 \\
1 \\
4\end{array}$ & $\begin{array}{l}7 \\
2 \\
4\end{array}$ & $\begin{array}{l}4 \\
2 \\
1\end{array}$ & $\begin{array}{l}3 \\
2 \\
5\end{array}$ & $\begin{array}{c}10 \\
4 \\
4\end{array}$ \\
\hline
\end{tabular}

As shown in Table 3, in the proposed scheme, the MU requires three modular exponentiations and five hash operations. As an example of the parameter length for security complexity, a 1024-bit prime $p$ and a 160-bit exponent $a$ can be adopted. In addition, two of three modular exponentiations can be pre-computed before starting the real session. The FA requires two exponentiations to establish the session key, generates one signature, verifies one signature, and performs one hash operation. The HA requires one exponentiation to authenticate the MU, generates one signature, verifies one signature, and performs four hash operations. It is easy to see that the proposed scheme is more efficient than existing schemes.

The proposed scheme uses no encryption and decryption primitives. There is a need to consider that the main cryptographic services of these schemes are intrinsically anonymous authentication and session key establishment between the MU and the FA. Therefore, in this phase, the important security functions are user authentication and message integrity between entities. Confidentiality can be achieved after establishing a common session key. As a result, encryption and decryption processing can be avoided in the proposed phase. Therefore, the proposed scheme is designed using only modular exponentiation, signature generation, signature verification, and hash functions.

Indeed, for the key exchange between two entities in the proposed scheme, the elliptic curve Diffie-Hellman protocol can be adopted instead of the DLP-based Diffie-Hellman protocol. However, in the elliptic curve Diffie-Hellman protocol, the HA generates elliptic curve domain parameters, the scalar point $P$, and its public key $C=c P$ and stores them in the smart card of the MU. Then the MU must always send these parameters with other messages to the FA in the authentication and session key establishment phase. 


\section{Conclusions}

This paper shows that Zhao et al.'s anonymous authentication scheme for roaming services in GLOMONET has weaknesses against a strong replay attack and is impractical for requiring the pre-distribution of system parameters. In addition, their scheme has unnecessary encryption/decryption operations that can increase of communications and computational costs. To overcome these weaknesses and inefficiencies, this paper proposes a novel anonymous authentication scheme that satisfies most security requirements and is suitable for low-power and resource-limited mobile devices based on its lightweight design.

\section{References}

[1] S. Suzukiz and K. Nakada, "An authentication technique based on distributed security management for the global mobility network", IEEE Journal Selected Areas in Communications, vol. 15, no. 8, (1997), pp. 1608-1617.

[2] C. Lee, M. Hwang, and I. Liao, "Security enhancement on a new authentication scheme with anonymity for wireless environments", IEEE Transactions on Industrial Electronics, vol. 53, no. 5, (2006), pp. 16831686.

[3] C. Wu, W. Lee, and W. Tsaur, "A secure authentication scheme with anonymity for wireless environments", IEEE Communications Letters, vol. 12, no. 10, (2008), pp. 722-723.

[4] D. He, M. Ma, Y. Zhang, C. Chen, and J. Bu, "A strong user authentication scheme with smart cards for wireless communications", Computer Communications, vol. 34, no, 3, (2011), pp. 367-374.

[5] J. Zhu and J. Ma, "A new authentication scheme with anonymity for wireless environments", IEEE Transactions on IEEE Communication Letter, vol. 13, no. 7, (2004), pp. 1118-1123.

[6] C. Chang, C. Lee, and Y. Chiu, "Enhanced authentication scheme with anonymity for roaming service in global mobility networks", Computer Communications, vol. 32, no. 4, (2009), pp. 611-618.

[7] T. Youn, Y. Park, and J. Lim, "Weaknesses in an anonymous authentication scheme for roaming service in global mobility networks", IEEE Communications Letters, vol. 13, no. 7, (2009), pp. 471-473.

[8] C. Li and C. Lee, "A novel user authentication and privacy preserving scheme with smart cards for wireless communications", vol. 55, no. 1-2, (2012), pp. 35-44.

[9] H. Mun, K. Han, Y. Lee, C. Yeun, and H. Choi, "Enhanced secure anonymous authentication scheme for roaming service in global mobility networks", Mathematical and Computer Modelling, vol. 55, no. 1-2, (2012), pp. 214-222.

[10] D. Zhao, H. Peng, L. Li, and Y. Yang, "A secure and effective anonymous authentication scheme for roaming service in global mobility networks", Wireless Personal Communications, vol. 78, no. 1, (2014), pp. 247-269.

[11] Y. Chen, S. Chuang, L. Yeh, and J. Huang, "A practical authentication protocol with anonymity for wireless access networks", Wireless Communications and Mobile Computing, vol. 11, no. 10, (2011), pp. 1366-1375

[12] Q. Xie, B. Hu, X. Tan, M. Bao, and X. Yu, "Robust anonymous two-factor authentication scheme for roaming service in global mobility network", Wireless Personal Communications, vol. 74, no. 2, (2014), pp. 601-614.

[13] A. Das, "A secure and effective user authentication and privacy preserving protocol with smart cards for wireless communications", Networking Science, vol. 2, no. 1-2, (2013), pp. 12-17.

[14] F. Wen, W. Susilo, and G. Yang, "A secure and effective anonymous user authentication scheme for roaming service in global mobility network", Wireless Personal Communications, vol. 73, no. 3, (2013), pp. 993-1004.

[15] W. Jeon, Y. Lee, and D. Won, "An efficient user authentication scheme with smart cards for wireless communications", International Journal of Security and Its Applications, vol. 7, no. 4, (2013), pp. 1-15.

[16] Z. Tan, "Cryptanalysis and improvements of an anonymous authentication scheme for roaming service in global mobility networks", International Journal of Security and Its Applications, vol. 7, no. 6, (2013), pp. 335-344.

[17] B. Hu, Q. Xie, M. Bao, and N. Dong, "Improvement of user authentication protocol with anonymity for wireless communications", Kuwait Journal of Science, vol. 41, no. 1, (2014), pp. 155-169.

[18] W. Stallings, Cryptography and Network Security: Principles and Practices, $3^{\text {rd }}$ ed. Prentice Hall, (2002)

[19] W. Diffie and M. Hellman, "New directions in cryptography", IEEE Transactions on Information Theory, vol. 22, no. 6, (1976), pp. 644-654.

[20] N. Koblitz, "Elliptic curve cryptosystem", Journal of Mathematics of Computation, vol. 48, no. 177, (1987), pp. 203-209. 


\section{Author}

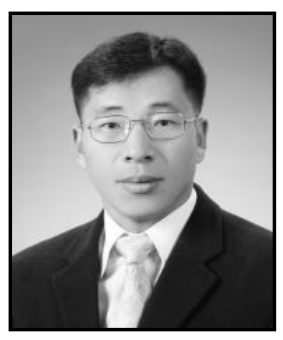

Jaecheol Ha, he received the $\mathrm{BE}, \mathrm{ME}$, and $\mathrm{PhD}$ in electronics engineering from Kyungpook National University, Rep. of Korea, in 1989, 1993, and 1998, respectively. He is currently a full professor of the department of information and security at Hoseo University, Asan, Rep. of Korea. During 1998 to 2006, he also worked as a professor in the department of information and communication at Korea Nazarene University, Cheonan, Korea. In 2006, he was a visiting researcher at the Information Security Institute of Queensland University of Technology, Australia. His research interests include network security, mobile security, smart card security, crypto chip design, and side-channel attacks. 